\title{
$Y$ chromosome microdeletions in idiopathic azoospermia and non-mosaic type of klinefelter syndrome
}

\author{
Yong Ho Lee', Tak Kim ${ }^{1}$, Mee Hye Kim², \\ Young Tae $\mathrm{Kim}^{1,3}$ and Sun Haeng Kim ${ }^{1}$ \\ ${ }^{1}$ Department of Obstetrics and Gynecology, College of Medicine, \\ Korea University Anam Hospital, Seoul 136-705, Korea \\ ${ }^{2}$ Department of Genetics, University of Glassgow, U.K. \\ ${ }^{3}$ Corresponding author: Tel, +82-2-920-5677; Fax, +82-2-926-5977; \\ E-mail, ytkim221@kuccnx.korea.ac.kr
}

Accepted 12 December 2000

Abbreviations: AZF, azoospermia factor; STS, sequence tagged site; IVF, in vitro fertilization; ICSI, intracytoplasmic sperm injection; $S R Y$, sex determining region $Y$; $C D Y$, chromodomain $Y$; $D B Y$, dead box $Y$; DFFRY, drosophilia fat facets related $Y$

\begin{abstract}
The objective of this study was to elucidate the cause of the spermatogenic defect in idiopathic azoospermia and non-mosaic type of Klinefelter syndrome. Genomic DNAs from 9 cases of Korean idiopathic azoospermia and 6 of Korean non-mosaic type of Klinefelter syndrome were used for the detection of $Y$ chromosome microdeletions by polymerase chain reaction using 60 primers. Microdeletions of the $Y$ chromosome were found in 1 of 9 (11.1\%) patients with idiopathic azoospermia, whereas none was deleted in non-mosaic type of Klinefelter syndrome. This result suggests that $Y$ chromosome microdeletions could be one of the etiologic factors in idiopathic azoospermia.
\end{abstract}

Keywords: $Y$ chromosome, microdeletion, idiopathic azoospermia, Klinefelter syndrome

\section{Introduction}

Approximately $15 \%$ of all couples attempting pregnancy experience failure of conception (Mosher WD, 1987). Male factor, either the sole cause or a contributing cause, has been identified in $50 \%$ of infertility cases (Bhasin et al., 1994; De Krester, 1997). Varicocele, infection, hypogonadism, cryptorchidism, structural abnormalities in reproductive organs, psychological problems, genetic defects and idiopathic infertility are encountered as causes of male infertility (Kim et al., 1997). Up to $66 \%$ of all infertile men have idiopathic azoospermia or severe oligospermia, depending on the definition of idiopathic that is no known causes (Hendry et al., 1973; Nieschlag, 1997). Since conventional cytogenetic technology cannot reveal any molecular defects of spermatogenesis in idiopathic azoospermia, microdeletions were used for molecular defects of $Y$ chromosome.

The role of $Y$ chromosome controlling spermatogenesis was first recognized in 1976 when partial deletions in the long arm of $Y$ chromosome were discovered (Tiepolo and Zuffardi, 1976). After dividing $Y$ chromosome into 7 regions (Vergnaud et al., 1986), the 3-nonoverlapping regions of $A Z F s$ ( $A Z F a, A Z F b, A Z F c$ ) have been mapped within intervals 5 and 6 of the $Y$ chromosome which lie within Yq11.21-Yq11.23. Although the large majority of microdeletions were located in the distal part (AZFc) with variable size of deletions (Reijo et al., 1995; Najmabadi et al., 1996; Qureshi et al., 1996; Stuppia et al., 1996; Vogt et al., 1996; Foresta et al., 1997; Pryor et al., 1997), men with deletions involving the more proximal parts (AZFa, AZFb) generally represent as azoospermic, and severe oligospermia may also be encountered in AZFb deletion.

Klinefelter syndrome is the most common chromosomal abnormality associated with male infertility and azoospermia. Its mechanism leading to spermatogenic defect is unknown.

The purpose of this study is to investigate the $Y$ chromosome microdeletions in idiopathic azoospermia and non-mosaic type of Klinefelter syndrome using polymerase chain reaction.

\section{Materials and Methods}

\section{Patients}

Nine cases of idiopathic azoospermia and six cases of non-mosaic type of Klinefelter syndrome from the Infertility Clinic of Korea University Anam Hospital were recruited for this study. Men of azoospermia secondary to infection, obstruction, and other causes of testicular injury are excluded. Azoospermia was confirmed with semen analysis according to the World Health Organization Guidelines (World Health Organization, 1993). As a control group 8 proven fathers were tested. This study was approved by Institutional Review Board and written informed consent was obtained from all patients.

\section{Genomic DNA preparation}

DNA extraction was performed by conventional method as follows. Briefly, $10 \mathrm{ml}$ of peripheral blood was collect- 
ed in EDTA bottles. RBC lysis buffer (12 mM Tris, $\mathrm{pH}$ 7.5; $5 \mathrm{mM} \mathrm{MgCl}_{2}$ ) was added to lymphocytes obtained from centrifugation. The resulting lymphocytes were supplemented with $20 \%$ sodium dodecyl sulfate, and proteinase $\mathrm{K}$ and were incubated overnight at $55^{\circ} \mathrm{C}$, following this $6 \mathrm{M} \mathrm{NaCl}$ was added. To precipitate DNA, 2 volumes of $99.5 \%$ ethanol was added and then the mixture was washed in $70 \%$ ethanol, and dissolved in distilled water. The concentration of DNA was measured by spectrophotometer at $260 \mathrm{~nm}$.

\section{PCR}

A set of $60 \mathrm{Y}$ specific STS (sequence tagged site) (accessed from Gene Bank, USA) was tested in each patient. The SRY, CDY, DBY and DFFRY gene and sY45, sY66, sY67, sY68, sY78, sY81, sY82, sY83, sY86, sY87, sY90, sY95, sY102, sY106, sY117, sY119, sY121, sY124, sY130, sY133, sY134, sY136, sY139, sY142, sY143, sY146, sY149, sY152, sY153, sY155, sY157, sY158, sY160, sY200, sY202, sY205, sY210, sY211, sY254, sY274, sY276, sY277, sY283, sY591, sY592, sY594, sY595, sY600, sY601, sY602, sY603, sY610, sY620, sY624, sY634, sY638 were used. Among them, sY205, sY254, sY277, sY283, sY624 were encoded in DAZ gene.

The polymerase chain reaction was performed with $100 \mathrm{ng}$ of genomic DNA in a final volume of $50 \mu \mathrm{l}$, including a reaction buffer, diethylnitrophenyl thiophosphate mix, each primer, and Taq polymerase. Thermocycling (Hybrid, UK) consisted of an initial denaturation of $5 \mathrm{~min}$ at $94^{\circ} \mathrm{C}$ and 35 cycles of $1 \mathrm{~min}$ at $94^{\circ} \mathrm{C}$ for denaturation, $1 \mathrm{~min}$ at $56^{\circ} \mathrm{C}$ for annealing, and $1 \mathrm{~min}$ at $72^{\circ} \mathrm{C}$ for extension, and then final extension of $10 \mathrm{~min}$ at $72^{\circ} \mathrm{C}$. In the sY121, sY160, sY211, sY254, sY283, sY592, sY610, sY638, the annealing temperature was $58^{\circ} \mathrm{C}$. The products were separated on $2 \%$ agarose gel by electrophoresis. And stained with ethidium bromide, and visualized using UV transillumination. A deletion was confirmed when two independent consecutive PCR amplifications yielded negative results.

\section{Results}

PCR screening of 60 loci in the $Y$ chromosome was performed in a group of patients with azoospermia who had idiopathic etiology $(\mathrm{N}=9)$ or Klinefelter syndrome $(\mathrm{N}=6)$, and 8 healthy fertile men as a control.

Deletion of $Y$ chromosome was found in 1 case of the $9(11.1 \%)$ cases with idiopathic azoospermia. The deleted sites were sY136 and sY277, and the 2 deleted sites were seen in the same patient (Figure 1 and Figure 2).

PCR was repeated in cases of deleted STSs, and the same results were obtained. There were no problems during amplification, since the positivity with sY136 and sY277 was observed in all other cases. The deleted sites were confined to the region of AZFb (sY136) and

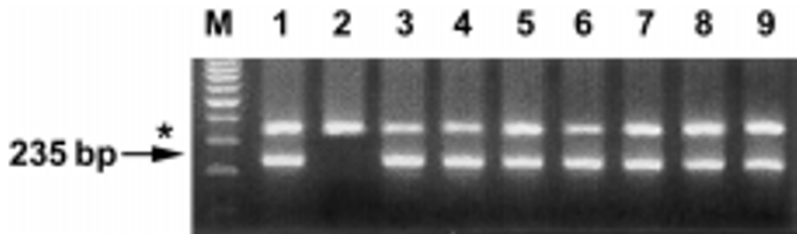

Figure 1. Deletion of sY136 in 1 of 9 patients with idiopathic azoospermia. Lane 2 shows deletion of sY136 corresponding to $235 \mathrm{bp}$. Primer sequence of SY136: Forward-CACATGAAGCACTGGAACTG, Reverse-GTTGTCTGGAAATCCCTGTG. M: marker. * : sY274 corresponding to $361 \mathrm{bp}$.

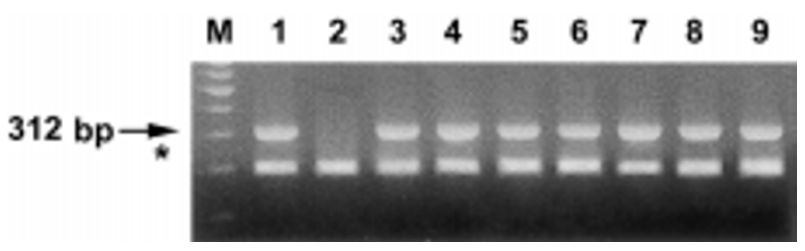

Figure 2. Deletion of sY277 in 1 of 9 patients with idiopathic azoospermia. Lane 2 shows deletion of sY277 corresponding to $312 \mathrm{bp}$. Primer sequence of SY277: Forward-GGGTTTTGCCTGCATACGTAATTA, Reverse-CCTAAAAGCAATTCTAAACCTCCAG. M: marker. * : sY276 corresponding to $216 \mathrm{bp}$

AZFc (sY277), which were limited to interval 6.

None of the 6 patients with azoospermic Klinefelter syndrome showed any deletion of the 60 loci tested, and no deletions were observed in any of the fertile men studied.

\section{Discussion}

Main function of $Y$ chromosome carrying about $1 \%$ of the human genome is the propagation of species through sex determination and control of spermatogenesis.

Defects of genes/loci on the $Y$ chromosome can influence the regulation of spermatogenesis (Bardoni et al., 1991; Ma et al., 1992; Vogt et al., 1992; Ma et al., 1993; Chandley et al., 1994; Kobayashi et al., 1994; Reijo et al., 1995; Kent-First et al., 1996; Najmabadi et al., 1996; Qureshi et al., 1996; Reijo et al., 1996; Stuppia et al., 1996; Vogt et al., 1996; Foresta et al., 1997; Mulhall et al., 1997; Pryor et al., 1997; Kim et al., 1999). Structure and function of the genes on the $Y$ chromosome to be elucidated is in progress, however the published data lead to the idea that microdeletions of the $Y$ chromosome could play a role in the step of spermatogenesis, partly.

Considering the microdeletions of $\mathrm{Y}$ chromosome, some questions concerning real frequency, indication of molecular diagnosis, and significance of the number and sites of primers investigated are raised.

The reported incidence of $Y$ deletions in men with azoospermia or severe oligospermia varies among studies, ranging from $1 \%$ (van der Ven et al., 1997) to $55 \%$ (Foresta et al., 1998); although most studies reported the incidence below $15 \%$. 
Microdeletions are found more frequently in azoospermic men than in oligozoospermic men (Simoni et al., 1998). The prevalence of microdeletions in azoospermic men was between the ranges of $6.7 \%$ (Kleiman et al., 1999) to $37.5 \%$ (Foresta et al., 1997). On the other hand, that in oligospermic men was between $1.5 \%$ (Oliva et al., 1998) to $22.7 \%$ (Foresta et al., 1997).

In our study, the frequency of microdeletion in idiopathic Korean azoospermia is $11.1 \%$ and it falls within the range reported previously.

Indication of molecular testing is not precisely defined at present. From the reports experienced to date in several centers, there has been an agreement with the fact that $Y$ chromosome analysis should be performed in all patients with a sperm concentration $<5 \times 10^{6} / \mathrm{ml}$. Molecular analysis would not help anatomical problems in case of varicocele and/or cryptorchidism, because molecular pathology in $\mathrm{Y}$ chromosome can also be found with these conditions (Pryor et al., 1997; Simoni et al., 1997). In addition, analysis of microdeletions should be recommended in all patients who are considering the use of IVF (in vitro fertilization) and ICSI (intracytoplasmic sperm injection).

To date, the question of which and how many loci should be analysed cannot be defined. The published data state that the frequency of microdeletions found is not dependent on the number of primers used. A higher number of STSs may lower inaccuracy, but very large numbers of primers to detect the microdeletions of $Y$ chromosome might be polymorphic variants and/or of clinical irrelevance.

This study used 60 primers to detect microdeletions but the deletion frequency was not increased compared to those of other reports. Microdeletions in infertile men, but not in their fertile fathers, suggested that the event occurred de novo in infertile patients (Ma et al., 1992; Reijo et al., 1995; Reijo et al., 1996) and it provided that the deletions were the cause of the spermatogenic failure observed in these men. Another possible explanation is gonadal mosaicism, the mosaic father might transmit the microdeletions to their sons. Of those who were not deleted in any region in germ-line DNA, their DNA from the testis tissue also might to be normal. To detect gonadal mosaicism, single cell analysis of spermatogenic cells may be required in patients considering the use of IVF \& ICSI.

The incidence of sex chromosome and autosomal anomalies revealed $4.2 \%$ and $1.5 \%$ of the azoospermic and oligospermic infertile men, respectively (Johnson, 1998). The predominate percentage of sex chromosome anomalies among infertile men was attributed to the Klinefelter syndrome $(47, \mathrm{XXY})$, which was the most common karyotypic anomaly to be found among infertile men. Azoospermia is almost the rule in men with Klinefelter syndrome who have the 47,XXY karyotype, although some studies have reported the presence of motile sperm in the ejaculate and testicular sperm in XY/ XXY mosaicism (Cozzi et al., 1994). To avoid the bias, we selected the group of Klinefelter syndrome that is non-mosaic type and azoospermia.

This study demonstrated that deletion was observed with a frequency of $11.1 \%$ using 60 STS primers in 9 patients with idiopathic azoospermia but not in 6 patients with Klinefelter's syndrome without spermatogenesis. This suggests that AZF deletion of $Y$ chromosome may contribute to the cause of idiopathic azoospermia, but different mechanisms of azoospermia in Klinefelter syndrome may exist. We suggested that over dosage of $X$ chromosome interferes with the function of $Y$ chromosome in non-mosaic type of Klinefelter syndrome.

\section{References}

Bardoni, B., Zuffardi, O., Guioli, S., Ballabio, A., Simi, P., Cavalli, P., Grimoldi, M. G., Fraccaro, M. and Camerino, G. (1991) A deletion map of the human Yq11 region: implications for the evolution of the Y-chromosome and tentative mapping of a locus involved in spermatogenesis. Genomics 11: 443-451

Bhasin, S., De Krester, D. M. and Baker, H. W. G. (1994) Clinical Review 64: Pathophysiology and natural history of male infertility. J. Clin. Endocrinol. Metab. 79: 1525-1529

Chandley, A. C. and Cooke, H. J. (1994) Human male fertility$\mathrm{Y}$ linked genes and spermatogenesis. Hum. Mol. Genet. 3: 1449-1452

Cozzi, J., Chevret, E., Rousseaux, S., Pelletier, R., Benitz, V., Jalbert, H. and Sele, B. (1994) Achievement of meiosis in XXY germ cells: study of 543 sperm karyotypes from an XY/ XXY mosaic patients. Hum Genet 93: 32-34

De Krester, D. M. (1997) Male infertility. Lancet 349: 787-790.

Foresta, C., Ferlin, A., Garolla, A., Rossato, M., Barbaux, S. and De Bortoli, A. (1997) Y-chromosome deletions in idiopathic severe testiculopathies. J. Clin. Endocrinol. Metab. 82: 1075-1080

Foresta, C., Ferlin, A., Garolla, A., Moro, E., Pistorello, M., Barbaux, S. and Rossato, M. (1998) High frequency of well defined $\mathrm{Y}$ chromosome deletions in idiopathic Sertoli cell only syndrome. Hum. Reprod. 13: 302-307

Hendry, W. F., Sommerville, I. F., Hall, R. R. and Pugh, R. C. B. (1973) Investigation and treatment of the subfertile male. Br. J. Urol. 45: 684-692

Johnson, M. D. (1998) Genetic risks of intracytoplasmic sperm injection in the treatment of male infertility: recommendations for genetic counseling and screening. Fertil. Steril. 70: 397-411

Kent-First, M. G., Kol, S., Muallem, A., Ofir, R., Manor, D., Blazer, S., First, N. and Itskovitz-Eldor, J. (1996) The incidence and possible relevance of $\mathrm{Y}$-linked microdeletions in babies born after intracytoplasmic sperm injection and their infertile fathers. Mol. Hum. Reprod. 2: 943-950

Kim, S. W., Kim, K. D. and Paick, J. S. (1999) Microdeletions within the azoospermia factor subregions of the $Y$ chromo- 
some in patients with idiopathic azoospermia. Fertil. Steril. 72 : 349-353

Kim, E. D. and Lipshultz, L. I. (1997) Advances in the evaluation and treatment of the infertile man. World J. Urol. 15: 378-393

Kleiman, S. E., Yogev, L., Gamzu, R., Hauser, R., Botchan, A., Lessing, J. B., Paz, G. and Yavetz, H. (1999) Genetic evaluation of infertile men. Hum. Reprod. 14: 33-38

Kobayashi, K., Mizuno, K., Hida, A., Komaki, R., Tomita, K., Matsushita, I., Namiki, M., Iwamoto, T., Tamura, S. and Minowada, S. (1994) PCR analysis of the Y-chromosome long arm in azoospermic patients: evidence for a second locus required for spermatogenesis. Hum. Mol. Genet. 3: 1965-1967

Ma, K., Sharkey, A., Kirsch, S., Vogt, P., Keil, R., Hargreave, T. B., McBeath, S. and Chandley, A. C. (1992) Towards the molecular localisation of the AZF locus: mapping of microdeletions in azoospermic men within 14 subintervals of interval 6 of the human Y-chromosome. Hum. Mol. Genet. 1: 29-33

Ma, K., Inglis, J. D., Sharkey, A., Bickmore, W. A., Hill, R. E., Prosser, E. J., Speed, R. M., Thomson, E. J., Jobling, M. and Taylor, K. (1993) A Y-chromosome gene family with RNAbinding protein homology: candidates for the azoospermia factor AZF controlling human spermatogenesis. Cell 75: 12871295

Mosher, W. D. (1987) Infertility: why business is booming. Am. Demogr. 9: $42-43$

Mulhall, J. P., Reijo, R., Alagappan, R., Brown, L., Page, D., Carson, R. and Oates, R. D. (1997) Azoospermic men with deletion of the DAZ gene cluster are capable of completing spermatogenesis: fertilization, normal embryonic development and pregnancy occur when retrieved testicular spermatozoa are used for intracytoplasmic sperm injection. Hum. Reprod. 12: $503-508$

Najmabadi, H., Huang, V., Yen, P., Subbarao, M. N., Bhasin, D., Banaag, L., Naseeruddin, S., de Kretser, D. M., Baker, H. W. and McLachlan, R. I. (1996) Substantial prevalence of microdeletions of the Y-chromosome in infertile men with idiopathic azoospermia and oligozoospermia detected using a sequence-tagged site-based mapping strategy. J. Clin. Endocrinol. Metab. 81: 1347-1352

Nieschlag, E. (1997) Classification of andrological disorders; in Nieschlag, E. and Behre, H. (ed): Andrology: Male Reproductive Health and Dysfunction. Berlin, Springer-Verlag, pp. 81-83

Oliva, R., Margarit, E. B. S., Ballesca, J. L., Carrio, A., Sanchez, A., Mila, M., Jimenez, L., Alvarez-Vijande, J. R. and Ballesta, F. (1998) Prevalence of $Y$ chromosome microdeletions in oligospermic and azoospermic candidates for intracytoplasmic sperm injection. Fertil. Steril. 70: 506-510

Pryor, J. L., Kent-First, M., Muallem, A., Van Bergen, A. H., Nolten, W. E., Meisner, L. and Roberts, K. P. (1997) Microdeletions in the Y-chromosome of infertile men. N. Engl. J. Med. 336: 534-539

Qureshi, S. J., Ross, A. R., Ma, K., Cooke, H. J., Intyre, M. A.,
Chandley, A. C. and Hargreave, T. B. (1996) Polymerase chain reaction screening for $\mathrm{Y}$-chromosome microdeletions: a first step towards the diagnosis of genetically-determined spermatogenic failure in men. Mol. Hum. Reprod. 2: 775-779

Reijo, R., Lee, T. Y., Salo, P., Alagappan, R., Brown, L. G., Rosenberg, M., Rozen, S., Jaffe, T., Straus, D. and Hovatta, O. (1995) Diverse spermatogenic defects in humans caused by Y-chromosome deletions encompassing a novel RNAbinding protein gene. Nature Genet. 10: 383-393

Reijo, R., Alagappan, R. K., Patrizio, P. and Page, D. C. (1996) Severe oligozoospermia resulting from deletions of azoospermia factor gene on Y-chromosome. Lancet 347: 1290-1293

Simoni, M., Gromoll, J., Dworniczak, B., Rolf, C., Abshagen, K., Kamischke, A., Carani, C., Meschede, D., Behre, H. M., Horst, J. and Nieschlag, E. (1997) Screening for deletions of the $\mathrm{Y}$ chromosome involving the DAZ gene in azoospermia and severe oligospermia. Fertil. Steril. 67: 542-547

Simoni, M., Kamischke, A. and Nieschlag, E. (1998) Current status of the molecular diagnosis of $Y$ chromosomal microdeletions in the work-up of male infertility. Initiative for international quality control. Hum. Reprod. 13: 1764-1768

Stuppia, L., Mastroprimiano, G., Calabrese, G., Peila, R., Tenaglia, R. and Palka, G. (1996) Microdeletions in interval 6 of the Y-chromosome detected by STS-PCR in 6 of 33 patients with idiopathic oligo-azoospermia. Cytogenet. Cell Genet. 72: 155-158

Tiepolo, L. and Zuffardi, O. (1976) Localization of factors controlling spermatogenesis in the nonfluorescent portion of the human Y chromosome long arm. Hum. Genet. 34: 119-124

van der Ven, K., Montag, M., Peschka, B., Leygraaf, J., Schwanitz, G., Haidl, G., Krebs, D. and van der Ven, H. (1997) Combined cytogenetic and $Y$ chromosome microdeletion screening in males undergoing intracytoplasmic sperm injection. Mol. Hum. Reprod. 3: 699-704

Vergnaud, G., Page, D. C., Simmer, M. C., Brown, L., Rouyer, F., Noel, B., Botstein, D., Chapelle, A. and Weissenbach, J. (1986) A deletion map of the human $Y$ chromosome based on DNA hybridization. Am. J. Hum. Genet. 38: 109-124

Vogt, P., Chandley, A. C., Hargreave, T. B., Keil, R., Ma, K. and Sharkey, A. (1992) Microdeletions in interval 6 of the Ychromosome of males with idiopathic sterility point to disruption of AZF, a human spermatogenesis gene. Hum. Genet. 89: 491-496

Vogt, P. H., Edelmann, A., Kirsch, S., Henegariu, O., Hirschmann, P., Kiesewetter, F., Kohn, F. M., Schill, W. B., Farah, S., Ramos, C., Hartmann, M., Hartschuh, W., Meschede, D., Behre, H. M., Castel, A., Nieschlag, E., Weidner, W., Grone, H. J., Jung, A., Engel, W. and Haidl, G. (1996) Human $\mathrm{Y}$-chromosome azoospermia factors (AZF) mapped to different subregions in Yq11. Hum. Mol. Genet. 5: 933-943

World Health Organization. Laboratory manual for the examination of human semen and semen-cervical mucus interaction. 3rd ed. New York: Cambridge University Press, 1993 